\title{
Análisis del sistema integral de recuperación del servicio en el sector hotelero de la ciudad de Cartagena
}

\author{
Analysis of the comprehensive recovery system of service in the hospitality industry \\ of the city of Cartagena
}

\section{Análise abrangente do sistema de recuperação de serviço no sector hoteleiro da cidade de Cartagena}

Mary Luz Estrada-Estrada ${ }^{1}$, Francisco José Arias-Aragonés², John Jairo Jurado-Coronell ${ }^{3}$

Forma de citar: M. L. Estrada-Estrada, F. J. Arias-Aragonés, J. J. Jurado-Coronell "Análisis del sistema integral de recuperación del servicio en el sector hotelero de la ciudad de Cartagena", Respuestas, vol. 21, no. 2, pp. 112-123, 2016.

Recibido:

Febrero 22 de 2016

Aceptado:

Mayo 27 de 2016

112

\section{Resumen}

Objetivo: La investigación se centró en analizar al sistema integral de recuperación del servicio desde la perspectiva del cliente interno. Metodología: Se tomó una muestra por conveniencia de 56 hoteles de la ciudad de Cartagena, en los cuales se aplicó entrevistas a expertos del sector y personal directamente relacionado con el servicio., Para el cálculo y análisis de los datos se utilizó el software SPSS. Resultados: Se encontró que los hoteles del sector aplican sistema integral de recuperación del servicio, pero existen unas oportunidades de mejoras dado que los promedios rodean entre 3.9 y 4,0 , los resultados más críticos se observan en la participación de los empleados en los mecanismos de recolección de la información disponible, participación de los empleados en el análisis de la información y la poca o nula capacitación para atender los fallos del servicio. Conclusiones: El sector hotelero de Cartagena necesita de procedimientos para recoger la información de los empleados referente a las deficiencias en el servicio, al igual que el involucramiento de todos los niveles de la organización en la identificación y análisis de la información. Además, el consenso en las acciones a tomar resulta determinante para entregar valor a los huéspedes y una respuesta rápida ayuda a generar la percepción en el cliente de que es tratado de manera justa por parte de la organización.

${ }^{2}$ Economista, Especialista en Ciencias Fiscales, Magister en Negocios Internacionales e Integración y Doctorando en

Economía y Empresa por la Universidad Castilla La Mancha (UCLM). Docente e Investigador franciscoarias100@hotmail.com. Orcid: 0000-0002-4543-0907 Fundación Universitaria Los Libertadores sede Cartagena. Cartagena-Colombia.

${ }^{3}$ Administrador de Empresas, Especialista en Gerencia de Proyectos, Magíster en Dirección Estratégica. Docente e investigador jjjuradoc21@hotmail.com. Orcid: 0000-0003-2607-7383 Fundación Universitaria Los Libertadores Sede Cartagena Cartagena - Colombia.
Palabras clave: Cliente interno, Fallos del Servicio, Fidelización, Recuperación del Servicio, Respuesta Rápida, SIRS.

\begin{abstract}
Objective: Research focused on analyzing the comprehensive recovery system of the service from the perspective of the internal client. Methodology: a sample was taken for convenience of 56 Hotels in the city of Cartagena, which applied interviews experts from the sector and staff directly related to the service, for the calculation and data analysis, the SPSS software was used. Results: Found that hotels in the sector apply comprehensive service recovery system, but there are a few opportunities for improvement given that surround the averages between 3.9 and 4,0, the most critical results can be seen in the participation of employees in the available data collection mechanisms, participation of employees in the analysis of information and little or no training for service failures. Conclusions: The hotel sector of Cartagena needs procedures for collecting information from employees regarding deficiencies in the service, as well as the involvement of all levels of the Organization in the identification and analysis of the information. Also, consensus
\end{abstract}


on the actions to take is decisive to deliver value to our guests and a rapid response helps to generate the perception on the client that is treated fairly by the organization.

Key words: recovery of the service, service failures, SIRS, quick response, internal customer.

\section{Resumo}

Objectivo: A investigação incidiu sobre a análise do sistema de recuperação de serviço integral a partir da perspectiva do cliente interno. Métodos: A amostra de conveniência de 56 hotéis na cidade de Cartagena, onde as entrevistas foi aplicado com especialistas da indústria e pessoal envolvido no serviço foi tomada, IIN o cálculo e análise de software SPSS dados foi utilizada . Resultados: Verificou-se que os hotéis do setor aplicam sistema abrangente de recuperação de serviço, mas existem algumas oportunidades de melhoria desde médias em torno de entre 3,9 e 4,0 , os resultados mais críticos são vistos na participação dos trabalhadores na mecanismos de recolha de informações disponíveis, a participação dos trabalhadores na análise de informações e pouco ou nenhum treinamento para lidar com falhas de serviço. Conclusões: A indústria hoteleira precisa de procedimentos de Cartagena para recolher informações de funcionários para as deficiências de serviço, bem como o envolvimento de todos os níveis da organização na identificação e análise de informações. Além disso, o consenso sobre as acções a tomar decisiva para entregar valor aos clientes e uma resposta rápida ajuda a gerar a percepção de que o cliente é tratado de forma justa pela organização.

Palavras-chave: falhas internas de atendimento ao público, lealdade, Recovery Service, de resposta rápida, SIRS.

\section{Introducción}

Es importante reconocer que en el proceso de la prestación del servicio es común que se cometan errores, pero es primordial que la solución de esto no se deje a la improvisación. Por eso, lo más aconsejable es tener planes y acciones previamente diseñados para enfrentar exitosamente estas situaciones. Las compañías que alcanzan niveles excepcionales en su atención desarrollan un sistema de recuperación de servicio basado en políticas y procedimientos que guían a los empleados para que puedan responder inmediatamente cuando el servicio falló y un cliente se siente molesto e insatisfecho. [1]

En la práctica, no todos los clientes van a estar satisfechos y pocas organizaciones pueden garantizar un servicio "cero error". Es inevitable el fracaso en el servicio y la insatisfacción de los clientes sobre todo en el sector del turismo debido a tres fuentes. En primer lugar, en la mayoría de los casos, la entrega del servicio se basa en la coordinación de la experiencia turística por los diferentes prestadores del servicio que puede aumentar la posibilidad del fracaso. En segundo lugar el turismo es un servicio basado en las personas y el impacto de la experiencia turística depende de un individuo en particular, lo cual aumenta potencialmente la ocurrencia de un fallo en el servicio y, en tercer lugar, la experiencia turística puede estar influenciada por factores externos no controlados por la organización como el clima, accesibilidad, entre otros. [2]

Con el propósito de dar respuestas a los fallos en el servicio y a la insatisfacción de los clientes, las compañías pueden desarrollar procesos de gestión de fallos a través de investigaciones, 
No. 2

Jul - Dic 2016 ISSN 0122-820X E-ISSN 2422-5053 PP: $112-123$ estrategias y buenas prácticas de recuperación del servicio., Debido a esto, desde mediados de los años noventa ha surgido una nueva línea de investigación que reclama una mayor atención a la información relativa a los fallos y a las oportunidades de aprendizaje que se desarrollan en las empresas de servicios [3]

Teniendo en cuenta los retos y avances de las organizaciones turísticas a nivel nacional e internacional en el tema de recuperación del servicio, se generan algunas preocupaciones y alertas en el sector hotelero de Cartagena, debido a que este ha sido un tema poco estudiado a nivel local y sobre el cual no se tiene la suficiente experiencia. Por eso, es apremiante mejorar la comprensión en esta área, debido a que la competencia por los mercados es cada vez mayor. En el actual contexto, se le exige al proveedor de servicios que se esfuerce por conseguir experiencias satisfactorias para sus clientes; es decir, un servicio sin fallos en la prestación [4].

Debido a la importancia que ha tomado en los últimos años esta línea de investigación y la necesidad de plantear soluciones a los problemas de competitividad en las empresas de la ciudad de Cartagena, resulta oportuno hacer un análisis de las estrategias de recuperación del servicio que aplican las empresas hoteleras de la ciudad, respecto al Sistema Integral de la Recuperación del Servicio (SIRS).

Actualmente existe una presión competitiva que exige al sector empresarial prestar los servicios de forma impecable. Sin embargo, aún en las mejores empresas, en ocasiones se presentan fallos que de no ser atendidos apropiadamente influyen negativamente en la percepción de los servicios de las empresas, por parte de los clientes. Dentro del marketing, los investigadores han planteado la denominada paradoja de la recuperación del servicio [5], [6], [7], la cual centra su atención en mostrar la importancia de recuperar el servicio como estrategia de fidelización de clientes, incluso logrando mejores resultados en este objetivo que cuando no se presenta ninguna falla.

En consecuencia a lo anterior, la investigación se convierte en referente para el análisis del servicio del sector turístico de diferentes entidades que desarrollan actividades en beneficio del sector. Además, plantea alternativas de mejora por medio de procesos de intervención (consultoría y capacitación), generando capacidades para el fortalecimiento competitivo del sector turístico como vocación de ciudad (región).

\subsection{Revisión de la literatura}

Las estrategias de recuperación del servicio consisten en la implementación de acciones específicas por parte de la empresa y/o sus empleados para rectificar, enmendar y compensar los inconvenientes o pérdidas experimentadas por los clientes como consecuencia de deficiencias en la prestación del servicio [8], [9]. Algunos ejemplos de fracaso del servicio son: producto no disponible, envío del producto equivocado, precio erróneo, producto defectuoso, lentitud en la prestación del servicio o tiempo de espera demasiado largo y comportamiento inadecuado de los empleados que venden el producto o prestan el servicio. Tales actividades de recuperación del servicio se han de concebir como un proceso planificado para restablecer la imagen de fiabilidad y calidad de la empresa, así como para reforzar la satisfacción de los clientes agraviados [10].

En el campo de la recuperación del servicio predomina un enfoque reactivo o pasivo, pues se centra en averiguar cómo los fallos y las correspondientes acciones correctoras influyen sobre el comportamiento del cliente [11], [12]. Este tipo de acciones correctoras, que corresponden a estrategias reactivas de recuperación del servicio, tienen únicamente una clara vocación de control de daños, dado que buscan reducir los niveles de insatisfacción del receptor del servicio, 
proporcionando una segunda oportunidad para que el cliente disfrute de una experiencia positiva, ayudando a mantener la lealtad de los clientes en cada caso particular [13], [14].

Existe una fase previa a la recuperación del servicio en la que la expectativa del cliente es importante en función de las características personales (lealtad, experiencias previas con la empresa, recomendaciones recibidas de terceros), la reputación de la empresa, las atribuciones por el fracaso en el servicio o el tipo y la severidad del fallo, entre otros. Luego le sigue la etapa de recuperación del servicio, una vez se ha detectado el fracaso, su origen, causas y personal implicado.

La estrategia de recuperación del servicio suele tener lugar en dos momentos de tiempo. En el primero, se soluciona el problema justo en el instante en que se produce el fracaso en el servicio, lo que corresponde a la estrategia proactiva [15]. En el segundo momento, se desarrollan estrategias de recuperación del servicio transcurrido un cierto tiempo posterior a la presencia del fracaso detectado por la empresa y/o por el cliente (estrategia reactiva) [11], [12].

El SIRS debe ofrecer la posibilidad de implementar ambos tipos de respuestas para que la empresa pueda anticipar y reaccionar de la manera más eficiente posible, ante la detección de un fallo en el servicio. [16]. Se señalan cuatro pasos clave: anticipar las necesidades de recuperación, actuar rápido, entrenar a los empleados y mantener informados a los consumidores sobre los cambios o mejoras introducidos en el servicio a raíz del problema detectado y corregido. Además, el SIRS plantea tres dimensiones o factores que son: detección de fallos, análisis de fallos y respuesta. Esta última dimensión está formada a su vez por cuatro factores: respuesta rápida, resultado justo, capacitación de los empleados y aprendizaje-innovación. Mediante todas estas dimensiones la empresa puede articular el enfoque estratégico, proactivo y relacional señalados, así como atender a la recuperación interna y externa de los fallos. Asimismo, este sistema conlleva un enfoque amplio del proceso fallorecuperación que combina una perspectiva externa e interna de sus implicaciones para la empresa [17].

La empresa cuenta tres fases de orientación al servicio, la operativa o acto de recuperación en sí mismo; la estratégica o aprendizaje derivado del análisis de los fallos; y la fase de visión de servicio, o transformación de la información o el conocimiento sobre los fallos en cambios y mejoras para la organización. [18]

La recuperación del servicio se distingue en tres resultados o consecuencias que los han denominado: recuperación del consumidor (clientes satisfechos), del proceso (procesos mejorados) y de los empleados (personal satisfecho). El conjunto de los tres resultados es lo que se denomina recuperación del servicio. La recuperación del consumidor y la del proceso están ligadas a las acciones de control de daños y la mejora de la prestación para evitar fallos futuros; coinciden pues con las fases de los trabajos previos [19].

Se ha estudiado la gestión de los fallos y su recuperación desde la perspectiva del cliente externo. Es decir, como afecta la recuperación del servicio en la satisfacción, imagen y fidelización del cliente en las empresas como un proceso. Sin que quede claro cuál es la percepción del cliente interno con respecto a la detección, análisis de los fallos, la capacidad de respuesta de los empleados y la resolución de problemas.

En este artículo se ha intentado superar las limitaciones de la literatura previa sobre la gestión de fallos y su recuperación desde la perspectiva del cliente interno con relación al Sistema Integral de Recuperación del Servicio (SIRS). 


\section{Materiales y métodos.}

En el presente estudio se hizo un análisis cuantitativo a partir del cuestionario con los directivos y con el personal que tiene mayor contacto con el cliente (huésped). El objetivo de estas entrevistas fue profundizar la comprensión de la relación de los colaboradores con la percepción que tienen sobre las estrategias que sigue el hotel para recuperación del servicio y por medio de esta información estructurar de manera más realista la investigación y resultados encontrados.
El trabajo de campo para esta investigación se desarrolló en hoteles ubicados en la zona turística de Cartagena de Indias entre el Centro Histórico y el barrio Bocagrande. Se entrevistaron 166 colaborados del área del servicio pertenecientes a 53 hoteles, entre marzo y julio de 2015. Los datos fueron analizados por el software estadístico SPSS versión 20.(Ver tabla I. Ficha técnica de la investigación).

Tabla I: Ficha técnica de la investigación

\begin{tabular}{|l|l|}
\hline Universo & Hoteles de la ciudad de Cartagena \\
\hline Ámbito geográfico & Cartagena de Indias, Colombia. \\
\hline Tamaño de la muestra & 166 \\
\hline Diseño de la muestra & Encuesta personal en lugar de trabajo. \\
\hline $\begin{array}{l}\text { Periodo de recogida de la } \\
\text { información }\end{array}$ & Marzo a Julio de 2015 \\
\hline Tipo de muestreo & Muestreo de conveniencia. \\
\hline Escala de medida & Likert \\
\hline
\end{tabular}

Fuente: Los autores

\section{Resultados y análisis.}

Como paso inicial del análisis de resultados se realizó la validación del instrumento de medida por medio del Alfa de Cronbach, para los cuales se obtuvieron en todos los casos valores superiores a 0,7 lo que indica que la escala es fiable. (Ver tabla II. Validación del instrumento).

Tabla II: Validación del instrumento

\begin{tabular}{|l|c|}
\hline \multicolumn{1}{|c|}{ FACTOR } & ALFA \\
\hline F1. DETECCIÓN DE FALLOS & 0.85 \\
\hline F2. ANÁLISIS DE FALLOS & 0.84 \\
\hline F3. RESPUESTA RÁPIDA. & 0.88 \\
\hline F4. RESPUESTA JUSTA & 0,72 \\
\hline F5. CAPACITACIÓN EMPLEADOS & 0,85 \\
\hline F6. INNOVACIÓN & 0,86 \\
\hline
\end{tabular}

Fuente: Los autores a partir del análisis de fiabilidad en el software SPSS

En los resultados obtenidos con respecto a la detección de fallos en el servicio por parte de los hoteles de la ciudad de Cartagena, con base en la tabla III y gráfica No.1, en general los hoteles tienen aceptables mecanismos para este proceso, dado que los promedios rondan alrededor de 4 puntos. Situación distinta sucede con los mecanismos de 
recolección de información disponibles para los empleados, los cuales presentan un promedio de 3,76 puntos, dato que permite mostrar la oportunidad de mejorar en los mecanismos de recolección de la información a los empleados. Tal como se ha planteado anteriormente, ellos son los receptores de la información sobre las quejas en servicio y su empoderamiento es condición necesaria en la recuperación del servicio.

Tabla III: Detección de fallos (DF)

\begin{tabular}{|c|l|}
\hline SIGLA & \multicolumn{1}{c|}{ PREGUNTA } \\
\hline DF1 & $\begin{array}{l}\text { Esta empresa dispone de procedimientos para recoger información de los empleados referente a } \\
\text { las quejas }\end{array}$ \\
\hline DF2 & Entregan encuestas de satisfacción a los clientes \\
\hline DF3 & $\begin{array}{l}\text { El sistema de recolección de información de quejas diseñados por la dirección son de fácil } \\
\text { acceso }\end{array}$ \\
\hline DF4 & Se dispone de procedimientos claros y explícitos para la recolección de quejas \\
\hline DF5 & $\begin{array}{l}\text { Cuando un cliente se encuentra con un fallo en la prestación del servicio se enfrenta a un } \\
\text { proceso sencillo para comunicarlo a la empresa }\end{array}$ \\
\hline
\end{tabular}

Fuente: Los autores a partir de las encuestas de recuperación del servicio en hoteles de Cartagena de Indias

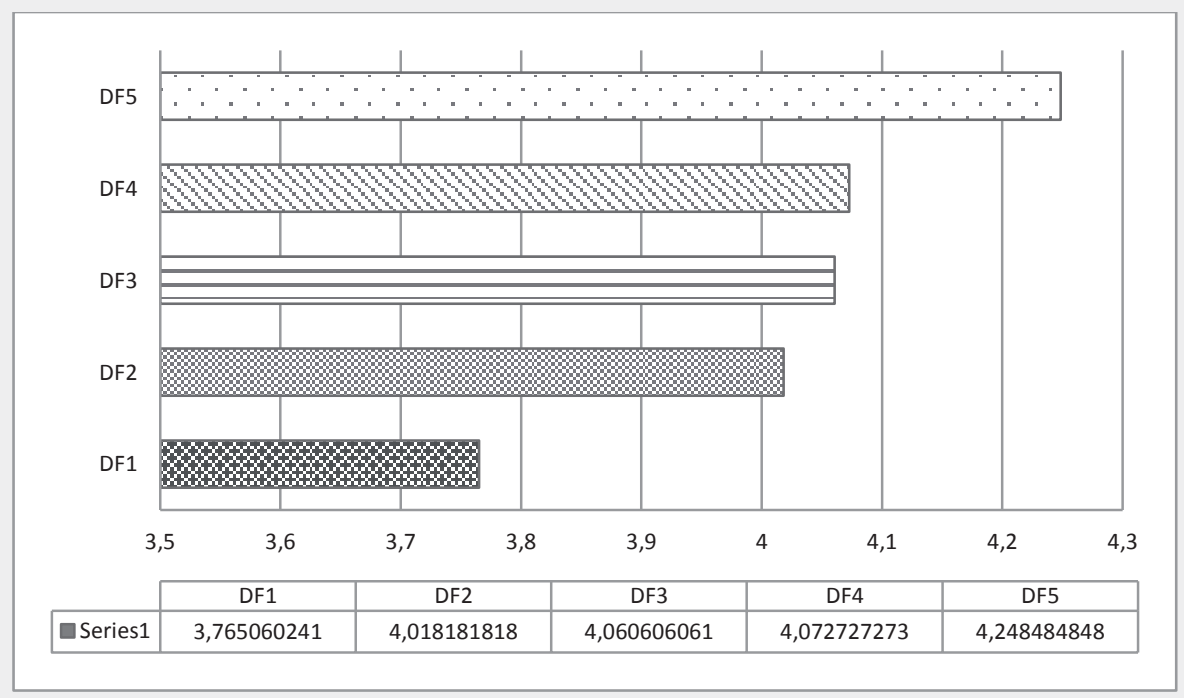

Gráfico1: Detección de Fallos

Fuente: Los autores a partir de las encuestas

La detección de fallos es un proceso medular en la entrega de valor; dar respuestas satisfactorias a los clientes cuando se han presentado fallos en el servicio, requiere identificar, compartir y analizar información de manera conjunta en todos los niveles, y tomar acciones sobre la recuperación en consenso [3]. En la tabla IV y grafico No.2 se muestran las oportunidades de mejora en los hoteles objeto de estudio, en varios de los indicadores del análisis de fallos, el promedio ronda el 4.1 puntos, en otros el 3.9. Los resultados más críticos se observan en la participación de los empleados en el análisis de la información recogida sobre los fallos para definir las prioridades de mejora del servicio y la participación en el análisis de los empleados en forma conjunta, en ambos los colaboradores no están de acuerdo y mucho menos en total acuerdo de que esta situación se presente en sus hoteles. (Promedio 3.9). Los colaboradores encuestados afirman estar de acuerdo en que en las organizaciones se rectifiquen los fallos en el servicio, se distribuya la información relevante sobre los problemas al personal y se analice detenidamente la información recogida. En esta parte, los empleados tienen el interés de escuchar los problemas de los clientes con el servicio a diferencia de lo planteado por: [].[19] 
No. 2

Jul - Dic 2016 ISSN 0122-820X E-ISSN 2422-5053
Tabla IV: Análisis de fallos (AF)

\begin{tabular}{|c|l|}
\hline SIGLA & \multicolumn{1}{c|}{ PREGUNTA } \\
\hline AF1 & Se distribuye rápidamente el problema detectado a todos los empleados \\
\hline AF2 & En la empresa se analiza detenidamente la información \\
\hline AF3 & El personal participa en el análisis de fallos dando prioridad a la mejora \\
\hline AF4 & El análisis de fallos identificados se realiza de manera conjunta \\
\hline AF5 & $\begin{array}{l}\text { Tras la detención de fallos la empresa evalúa como rectificar o mejorar los procesos de prestación del } \\
\text { servicio. }\end{array}$ \\
\hline
\end{tabular}

Fuente: Los autores a partir de las encuestas de recuperación del servicio en hoteles de Cartagena de Indias

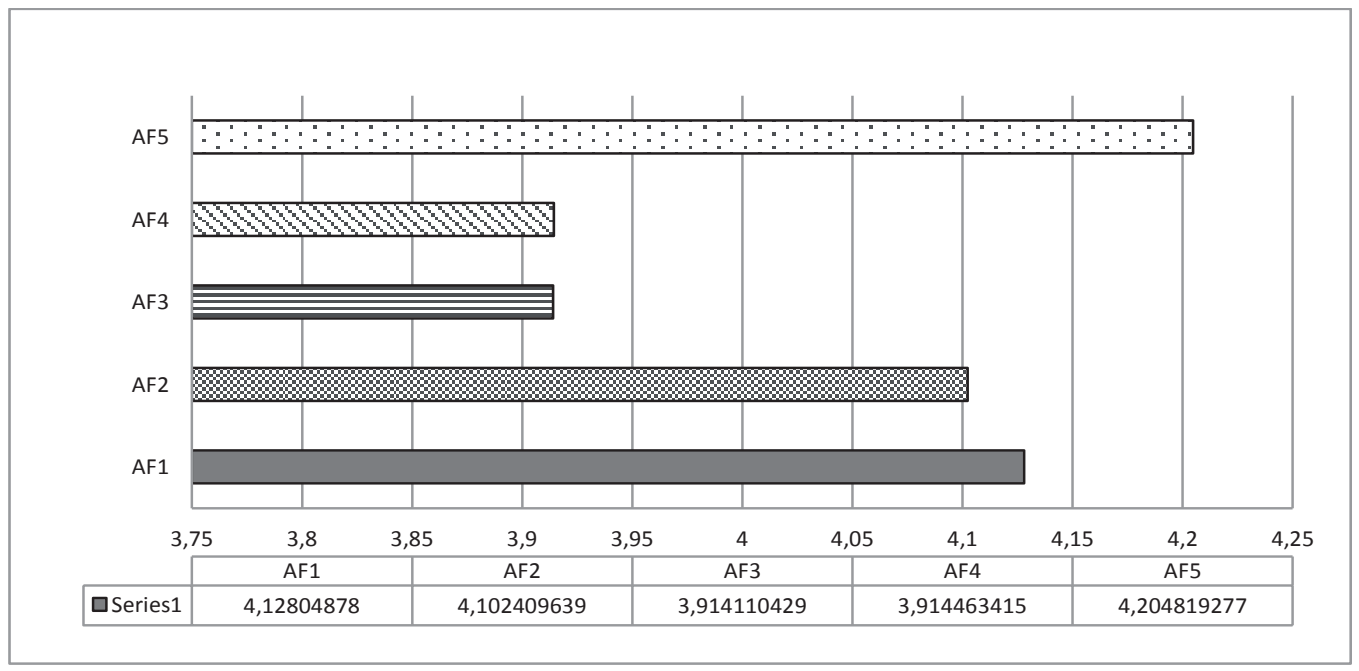

Gráfico 2: Análisis de fallos

Fuente: Los autores a partir de las encuestas de recuperación del servicio en hoteles de Cartagena de Indias
Para analizar los fallos en los hoteles encuestados se determinaron 5 preguntas (Tabla IV: análisis de fallos) que ayudó a identificar los siguiente:

Los clientes no solo esperan la recuperación del servicio, sino también recompensas que van desde la devolución del dinero, entrega de descuento y cambios de productos, acciones que son importantes para el mantenimiento y la fidelización de los clientes.

Es importante tener en cuenta que no solo con 118
[20]. Diferentes investigaciones proponen desglosar la respuesta orientada al cliente en tres factores: respuesta rápida, resultado justo y capacitación de personal. La proximidad de la solución con el problema es vital para no afectar la satisfacción y experiencia del cliente, las respuestas que son percibidas como justas, por lo general guardan estrecha relación con la rapidez en que se solucionan [21].

La información recolectada para el análisis descriptivo, tal como se aprecia en la tabla $\mathrm{V}$ y gráfica No. 3, muestra que los encuestados manifestaron estar de acuerdo en que el hotel de respuestas rápidas a los fallos presentados, rectificar el servicio a la mayor brevedad $(4,3)$, responder ante los fallos del servicio inmediatamente $(4,4)$, dar respuesta rápida al cliente $(4,4)$, responder a tiempo a la gestión de las quejas $(4,2)$, los anteriores son indicadores evaluados con promedios superiores a cuatro $(4,0)$, la más alta evaluación de todas dimensiones analizadas hasta el momento. 


\section{Mary Luz Estrada-Estrada, Francisco José Arias-Aragonés, Johr}

Tabla V: Respuesta rápida de la empresa

\begin{tabular}{|c|c|}
\hline SIGLA & \multicolumn{1}{c|}{ PREGUNTA } \\
\hline RPR1 & El empleado está preparado para asumir su responsabilidad ante una falla \\
\hline RPR2 & La gestión de las quejas se efectúan a tiempo \\
\hline RPR3 & Los fallos en la prestación de servicio son solventados de forma rápida \\
\hline RPR4 & Para esta empresa es prioridad responder inmediatamente el fallos \\
\hline RPR5 & La empresa rectifica a la mayor brevedad el servicio fallido \\
\hline
\end{tabular}

Fuente: Los autores a partir de las encuestas de recuperación del servicio en hoteles de Cartagena de Indias
Cúcuta-Colombia

Vol. 21

No. 2

Jul - Dic 2016

ISSN 0122-820X

E-ISSN 2422-5053

PP: 112-123
La tabla $\mathrm{V}$ permitió determinar que un cliente que se vea afectado por una falla en el servicio, es probable que primero acuda a un empleado o representante de servicio de la empresa, quien debe estar en capacidad y disposición de resolver de forma apropiada y satisfactoria, ante la falla en el servicio. Un colaborador capacitado gana confianza, convirtiendo la molestia en complacencia [22]. La capacitación perfila a los colaboradores del servicio para una mayor autonomía y empoderamiento en la solución de problemas con los clientes, sobre todo en aquellas situaciones poco comunes, las cuales a lo mejor no están previstas en los manuales de procedimientos y en otros documentos corporativos.

La grafica 3 enseña los resultados de los hoteles al resolver y dar respuesta rápida y positiva a los problemas presentados en la prestación del servicio., Se aprecia que el promedio es superior a 4, donde la rectificación adecuada y oportuna se hace evidente por parte de todo el personal a cargo; teniendo como prioridad la atención de las quejas y búsqueda de la satisfacción total del cliente.

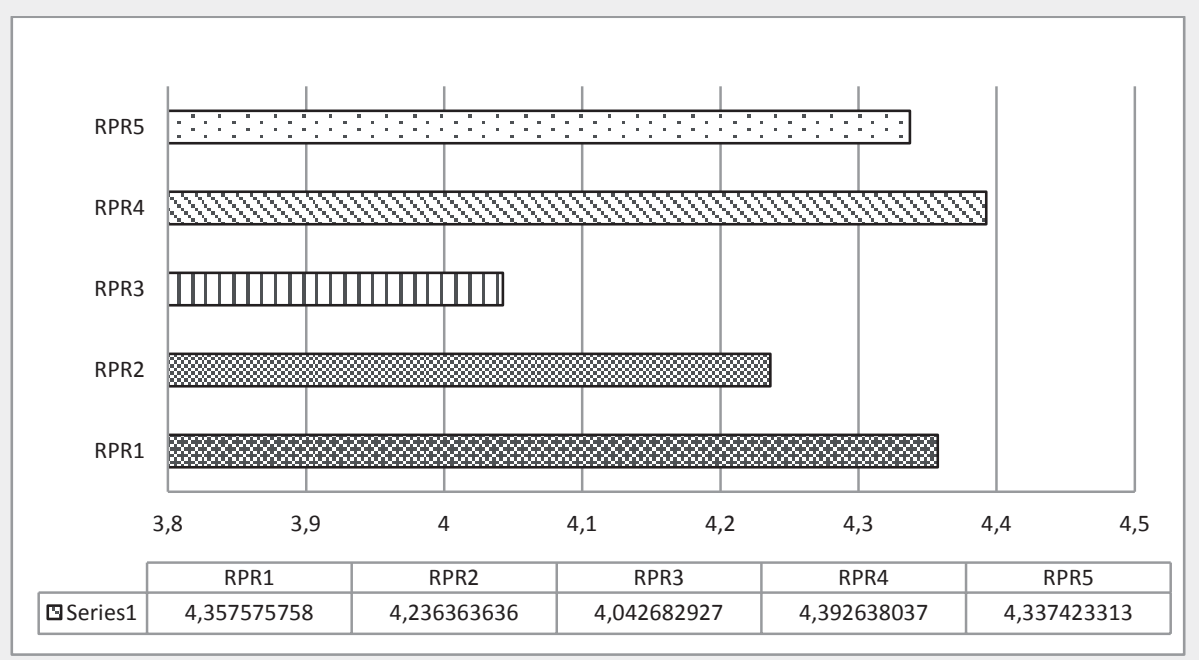


La capacitación es un principio básico de la gestión de calidad y es responsabilidad de todos los miembros de la organización, de manera tal que los empleados puedan contar con niveles mínimos de responsabilidades $\mathrm{y}$ de atribuciones para detectar y solucionar problemas [23] más cuando se trata de personal en contacto con el cliente porque deben de estar preparados para actuar frente a cualquier situación y poder de manera responsable asumir los problemas, dando así autonomía y confianza y permitiendo ampliar sus conocimientos.

En la tabla VI y grafico 4 se observa que los colaboradores en un promedio de 3.8, lo cual sugiere que no tienen la mejor formación para atender o solucionar problemas y les falta más libertad para rectificar el fallo y dar respuesta rápida (3.9).

Tabla VI: Capacitación a empleados

\begin{tabular}{|c|l|}
\hline SIGLA & \multicolumn{1}{c|}{ PREGUNTA } \\
\hline CE1 & Los empleados tiene poder para rectificar libremente \\
\hline CE2 & Confian en la capacidad de decisión de los empleados \\
\hline CE3 & La dirección permite a sus empleados tomar decisiones oportunas para rectificar el fallo. \\
\hline CE4 & Los empleados reciben información específica para darle solución a los problemas \\
\hline CE5 & Los empleados están capacitados y reciben capacitación para gestionar las respuestas rápidas \\
\hline
\end{tabular}

Fuente: Los autores a partir de las encuestas de recuperación del servicio en hoteles de Cartagena de Indias

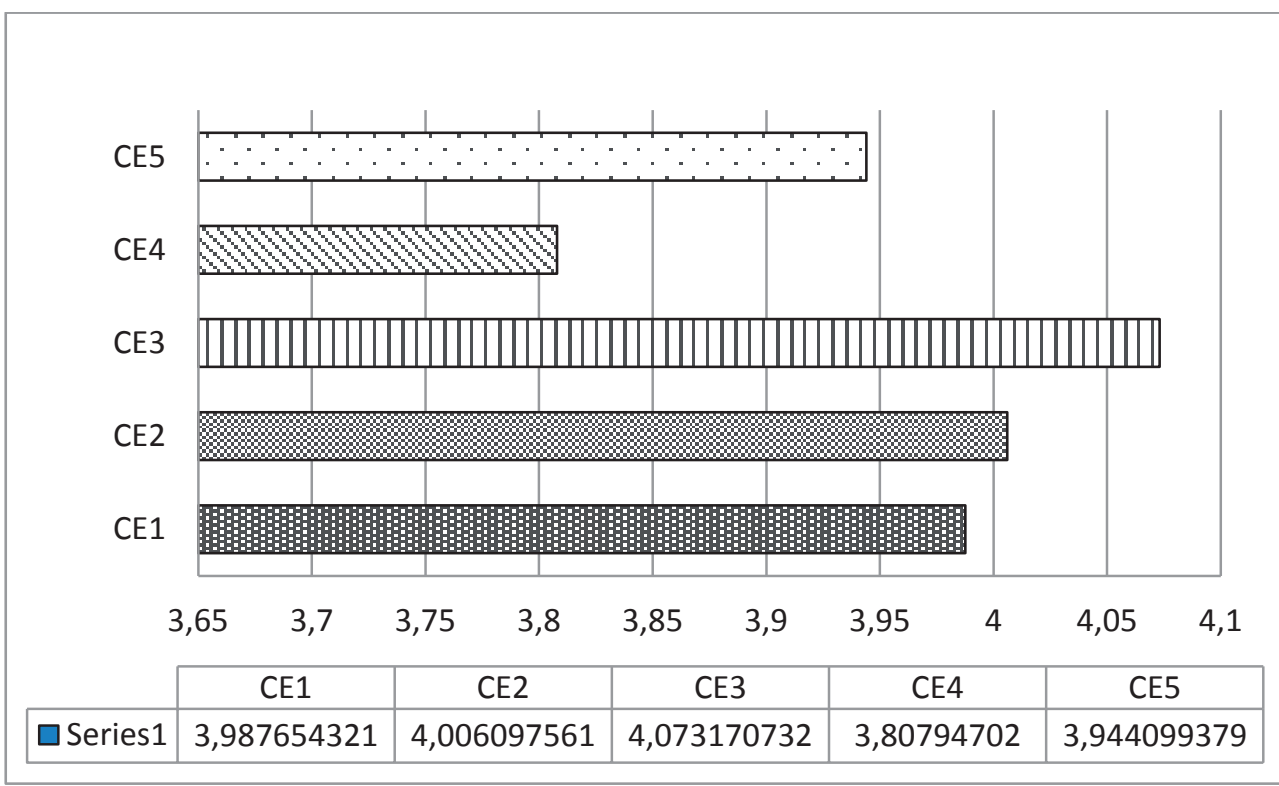

Gráfico 4: Capacitación a empleados

Fuente: Los autores a partir de las encuestas de recuperación del servicio en hoteles de Cartagena de Indias

La tabla IV permitió determinar que para las empresas es necesario conocer los fallos que se han producido en la prestación del servicio a los clientes y que dichos fallos se puedan anticipar, por lo que se debe desarrollar procedimientos específicos para obtener este tipo de información. Este tipo de prácticas entendemos que permiten anticipar 
la recuperación del proceso antes de que se produzca el fallo, conocer con prontitud la necesidad de recuperación del cliente y facilitar su recuperación inmediata. [17]

\section{Conclusiones}

A pesar de que la recuperación del servicio es prioridad a la hora de gestionar estrategias dentro de los hoteles de la ciudad de Cartagena, hace falta mayor comprensión y preparación en el tema. Aunque los hoteles hacen esfuerzos por implementar procesos que conduzcan a la recuperación del servicio se presentan dificultades en los temas de participación de los empleados en los mecanismos de recolección y análisis de la información sobre los fallos, adicionalmente se encontró evidencia de la necesidad de capacitar al personal para actuar correctamente ante la ocurrencia un fallo y hacer la recuperación del servicio.

En los hoteles de 2 y 3 estrellas se encontró un sistema débil de recolección de información, con muchas dificultades para mantener al cliente satisfecho y fiel a sus servicios; esto sucede por los limitados recursos de los cuales disponen, muy a pesar de que la administración conoce las ventajas de un sistema de recuperación de servicio. Por tal razón, se establecen estrategias desacertadas que no logran hacer una adecuada y efectiva recuperación del servicio.

Para lograr el éxito de toda actividad o proceso se debe incluir el personal en su totalidad, ya que el cliente interno es tan importante como el externo, siendo éste el que ejecuta la actividad y representa a la empresa; pero a pesar de esto, en algunos hoteles el empleado no está siendo involucrado en las actividades, no le es reconocido por su desempeño, no se le motiva para que además de ir a trabajar se sienta feliz de hacerlo, no se tiene en cuenta al momento de iniciar con procesos nuevos, que para ellos, pueden significar un choque, puesto que hacían las cosas de otra manera y todo cambio trae consigo cierto nivel de resistencia. Lo anterior forma parte de la estrategia de recuperación del servicio, dado que la motivación en el trabajador genera un mayor compromiso y responsabilidad, lo cual es trasmitido a los clientes, garantizando el logro de los objetivos.

Los hoteles como todas las empresas deben diseñar estrategias de recuperación del servicio para aumentar el flujo de clientes y para mantener un servicio de calidad, involucrando a todo el personal en el logro y objetivo común de fidelizar e ir más allá de satisfacer las necesidades y expectativas de los clientes.

Para finalizar y resumiendo algunas ideas, no queda duda de que el sector hotelero necesita de procedimientos para recoger la información de los empleados referente a las deficiencias en el servicio, al igual que el involucramiento de todos los niveles de la organización en la identificación y análisis de la información. Además, el consenso en las acciones a tomar resulta determinante para entregar valor a sus huéspedes y una respuesta rápida ayuda a generar la percepción en el cliente de que es tratado de manera justa por parte de la organización.

\section{Referencias}

[1] M. Robleda, C. Bruno, E. Centurión and S. Dzul. "Medición de la calidad en el servicio en Hoteles de Celestún, Yucatán". Revista Ecorfan, vol 5, no 12, pp.197-211, 2014.

[2] D. Grewal, M. Levy and V. Kumar. "Customer experience management in retailing: an organizing framework", Journal of Retailing, vol. 85, no.1, pp. 1-14, 2009.

[3] S. Tax and S. Brown. "Recovering and Learning from Service Failure", Sloan Management Review, vol. 40, no. 1, pp. 75-88, 1998. 
[4] P. Patterson, E. Cowley and K. Prasongsukarn. "Service failure recovery: the moderating impact of individual-level cultural value orientation on perceptions of justice". International Journal of Research in Marketing, vol. 23, no. 3, pp. 263-277, 2006.

[5] M. J, Bitner. "Evaluating service encounters: the effects of physical surroundings and employee responses". The Journal of Marketing, vol. 64, no. 2, pp. 69-82, 1990.

[6] M.A. Mccollough, and S. G. Bharadwaj. "The recovery paradox: an examination of consumer satisfaction in relation to disconfirmation, service quality, and attribution based theories", Marketing theory and applications, vol. 3 pp.119, 1992.

[7] V. A. Zeithaml, L. L. Berry and A. Parasuraman. "The behavioral consequences of service quality". Journal of Marketing, Vol. 60, no. 2, pp. 31-46, 1996.

[8] S.W. Kelley, y M.ADavis. “Antecedents to customer expectations for service recovery", Journal of the Academy of Marketing Science, vol. 22, no. 52, pp. 52-61, 1994.

[9] N. J Ashill, J. Carruthers and J. Krisjanous. "Antecedents and outcomes
[11] K. L Reynolds and L.C Harris, "Dysfunctional customer behavior severity: an empirical examination", Journal of Retailing, vol. 85, no. 3, pp. 321-335, 2009.

[12] J. Wirtz and A.S Mattila. "Consumer responses to compensations, speed of recovery and apology after a service failure", International Journal of Service Industry Management, vol. 15, no. 2, pp. 150-166, 2004.

[13] K. Schoefer and C. Ennew. "The impact of perceived justice on consumer emotional responses to service complaints experiences", Journal of Services Marketing, Vol. 19, no. 5, pp. 261-270, 2005.

[14] A. K. Smith, and R. N. Bolton. "The effect of customers' emotional responses to service failures on their recovery effort evaluations and satisfaction judgments", Journal of the Academy of Marketing Science, vol. 30, no. 1, pp.523, 2002.

[15] O. Hart. "Is" Bounded Rationality" an Important Element of a Theory of Institutions?", Journal of Institutional and Theoretical Economics (JITE)/Zeitschrift für die gesamte Staatswissenschaft, vol. 146, no. 4, pp. 696-702, 1990.

[16] M. L. Santos Vijande, A. M. Díaz Martín, L. Suárez Álvarez, and A. B. Del Río Lanza. "Configuración de un Sistema Integral de Recuperación del Servicio (SIRS): aplicación en empresas de servicios industriales". Información Comercial Española, ICE: Revista de economía, no. 856, pp.103-122, 2010.

[17] K. V. La, and J. Kandampully. "Market oriented learning and customer value enhancement through service recovery management", Managing Service 
Quality: An International Journal, vol. 14, no.5, pp. 390-401, 2004.

[18] H. R. Johnston and M. R. Vitale.

"Creating Competitive Advantage with Interorganizational Information Systems" MIS Quarterly, vol. 12, no.2, pp. 153-165, 1998.

[19] C. Homburg and A. Fürst. "See no evil, hear no evil, speak no evil: a study of defensive organizational behavior towards customer complaints", Journal of the Academy of Marketing Science, vol. 35, no. 4, pp. 523-536, 2007.

[20] H. Koontz and H. Weihrich. "Administración global, comparada y de calidad". Koont M, Weihrich H. Administración: Una perspectiva global. 11a Edición México: McGrawHill Interamericana, 1998, pp. 81-120.

[21] S. S. Tax, S.W. Brown and M. Chandrashekaran. "Customer evaluations of service complaint experiences: implications for relationship marketing", Journal of Marketing, vol. 62, no. 2, pp. 6076, 1998.

[22] R. Zemke, T.R Bacon and C.R Bell. Knock your socks off service recovery. AMACOM Div American Mgmt Assn, 2000.

[23] T. Dewitt and M.K Brady. "Rethinking service recovery strategies the effect of rapport on consumer responses to service failure", Journal of Service Research, vol. 6, no.2, pp. 193-207, 2003. 